\title{
Fraglicher Nutzen
}

\section{Hier steht eine Anzeige.}

Fragstellung: Ist bei Patienten mit einer symptomatischen Stenose der Arteria vertebralis die Angioplastie mit Stenting einer rein medikamentösen Therapie überlegen?

Hintergrund: Für die Therapie symptomatischer Karotisstenosen belegten mehr als zehn Jahre zurückliegende Studien, dass die Karotisendarterektomie einer medikamentösen Behandlung überlegen ist. In der Folgezeit konnte dann gezeigt werden, dass Karotisoperationen und Stenting vergleichbare Ergebnisse erzielen, wobei das Komplikationsrisiko des Stentings bei über 70-jährigen Patienten erhöht ist. Es liegen allerdings keine neuen Studien vor, welche die interventionelle Therapie mit einer optimalen und intensiven medikamentösen Behandlung verglichen hätten. Die VIST-Studie (Vertebral Artery Ischaemia Stenting Trial) ging nun der Frage nach, ob bei Patienten mit einer symptomatischen Vertebralisstenose eine Angioplastie plus Stenting einer bestmöglichen medikamentösen Behandlung überlegen ist.

Patienten und Methodik: Es handelt sich um eine prospektive, randomisierte, offene Studie, die an 14 Krankenhäusern in Großbritannien durchgeführt wurde. Die Patienten hatten eine symptomatische Vertebralisstenose von mehr als $50 \%$ Lumeneinengung und wurden entweder interventionell mit Angioplastie und Stenting behandelt, plus bestmöglicher medikamentöser Therapie, oder nur bestmöglich medikamentös. Die Studie musste wegen mangelnder Rekrutierung nach 182 Patienten abgebrochen werden.

Ergebnisse: Für die Auswertung standen in der Stentinggruppe 91 und in der medikamentösen Therapiegruppe 88 Patienten zur Verfügung. Diese waren im Mittel 67 Jahre alt. $75 \%$ der Patienten waren Männer. Zwei Drittel der Patienten hatten einen Schlaganfall und ein Drittel eine transitorische ischämische Attacke (TIA) erlitten. Im Mittel vergingen in der Stentinggruppe 16 Tage zwischen Ereignis und Stenting. Mehr als $80 \%$ der Stenosen waren extrakraniell lokalisiert. Der primäre Endpunkt umfasste tödliche und nicht tödliche Schlaganfälle. Er trat bei fünf der Patienten in der Stenting- und bei zwölf Patienten in der Medikamentengruppe auf. Dies entspricht einer Hazard Ratio (HR) von 0,40, die mit einem p-Wert von 0,08 nicht signifikant war. Die HR für Schlaganfall und TIA betrug 0,50 mit einem $\mathrm{p}$-Wert von 0,05.

Markus HS, Larsson SC, Kuker W et al. Stenting for symptomatic vertebral artery stenosis: the vertebral artery ischaemia stenting trial. Neurology 2017; 89: $1229-36$
Schlussfolgerungen: Das Stenting von extrakraniellen Vertebralisstenosen hat eine geringe Komplikationsrate. Der therapeutische Nutzen wurde durch diese Studie allerdings nicht belegt.

\section{- Kommentar von Hans-Christoph Diener, Essen}

\section{Eine Phase-III-Studie ist unwahrscheinlich}

Die in Großbritannien durchgeführte Studie hat ein ähnliches Schicksal erlitten wie andere Studien, bei denen eine interventionelle Therapie mit bestmöglicher medikamentöser Therapie verglichen wurde, nämlich ein vorzeitiges Studienende wegen mangelnder Rekrutierung. Diese Studie zeigt einen Trend zugunsten der Intervention, wobei dieser aber angesichts der geringen Patientenzahl nicht signifikant wurde. Ob es jemals möglich sein wird, wie von den Autoren gefordert, eine größere Phase-III-Studie durchzuführen, ist zweifelhaft. 